Kennesaw State University

DigitalCommons@Kennesaw State University

Faculty Publications

$12-1987$

\title{
Some Applications of the Bounded Convergence Theorem for an Introductory Course in Analysis
}

Jonathan W. Lewin

Kennesaw State University, jlewin@kennesaw.edu

Follow this and additional works at: http://digitalcommons.kennesaw.edu/facpubs

Part of the Mathematics Commons

\section{Recommended Citation}

Lewin, J. W. (1987). Some applications of the bounded convergence theorem for an introductory course in analysis. The American Mathematical Monthly, 94(10), pp. 988-993.

This Article is brought to you for free and open access by DigitalCommons@Kennesaw State University. It has been accepted for inclusion in Faculty Publications by an authorized administrator of DigitalCommons@Kennesaw State University. For more information, please contact 


\title{
Some Applications of the Bounded Convergence Theorem for an Introductory Course in Analysis
}

\author{
JONATHAN W. LEWIN \\ Kennesaw College, Marietta, GA 30061
}

The Arzela bounded convergence theorem is the special case of the Lebesgue dominated convergence theorem in which the functions are assumed to be Riemann integrable.

The Bounded Convergence Theorem. Suppose $\left(f_{n}\right)$ is a sequence of functions which are Riemann integrable on an interval $[a, b]$, suppose that the sequence $\left(f_{n}\right)$ converges pointwise to a function $f$, and suppose that there exists a number $K$ such that $\left|f_{n}(x)\right| \leqslant K$ for all $n \in \mathbb{Z}^{+}$and $x \in[a, b]$. Then the sequence of integrals $\int_{a}^{b} f_{n}(x) d x$ converges, and in the event that the function $f$ is also Riemann integrable on $[a, b]$, we have

$$
\int_{a}^{b} f_{n}(x) d x \rightarrow \int_{a}^{b} f(x) d x .
$$

Because the proof of this theorem has traditionally been perceived as quite hard, or dependent on concepts which lie beyond a first course in analysis, the theorem is presently omitted in such courses, and its applications at this level have therefore been somewhat neglected. However, a recent paper [3] of the author shows that the bounded convergence theorem can be proved quite easily in a first course, and it is therefore worth knowing what its applications might be. In this paper we shall show how the bounded convergence theorem may be used to obtain simple proofs of some quite sharp forms of the theorems which concern differentiation under the integral sign and inversion of repeated integrals. We shall obtain versions of these theorems which are distinctly sharper than the results usually found in an undergraduate text.

Differentiation under the integral sign. In a typical first course in analysis, the theorems on differentiation under the integral sign are given for continuous functions only (see, for example, Buck [2] Theorems 10 and 29, or Apostol [1], Theorem 7.40). However, using the bounded convergence theorem, it is easy to drop the requirement of continuity, and obtain sharper theorems of the type one might expect to see at a more advanced level using Lebesgue integrals. A theorem of the sharper type may be found in [1, Theorem 10.39], three chapters beyond Theorem 7.40 , in the chapter on Lebesgue integration.

Theorem on Differentiating Under the Integral Sign. Suppose $f:[a, b] \times$ $S \rightarrow \mathbb{R}$, where $S \subseteq \mathbb{R}$, and that for every point $y \in S$, the Riemann integral

$$
\phi(y)=\int_{a}^{b} f(x, y) d x
$$

exists. Suppose $y_{0}$ is both a point of $S$ and a limit point of $S$, and that for every 
$x \in[a, b]$, the partial derivative

$$
D_{2} f\left(x, y_{0}\right)=\lim _{y \rightarrow y_{0}}\left[\frac{f(x, y)-f\left(x, y_{0}\right)}{y-y_{0}}\right]
$$

exists, and suppose that the Riemann integral $\int_{a}^{b} D_{2} f\left(x, y_{0}\right) d x$ exists. Suppose finally that there exists a number $K$ such that for all $x \in[a, b]$ and $y \in S \backslash\left\{y_{0}\right\}$, we have

$$
\left|\left[\frac{f(x, y)-f\left(x, y_{0}\right)}{y-y_{0}}\right]\right| \leqslant K \text {. }
$$

Then

$$
\phi^{\prime}\left(y_{0}\right)=\int_{a}^{b} D_{2} f\left(x, y_{0}\right) d x
$$

Proof. We deduce this theorem directly from the bounded convergence theorem. Given any sequence $\left(y_{n}\right)$ in $S \backslash\left\{y_{0}\right\}$, converging to $y_{0}$, we have

$$
\frac{\phi\left(y_{n}\right)-\phi(y)}{y_{n}-y}=\int_{a}^{b}\left[\frac{f\left(x, y_{n}\right)-f\left(x, y_{0}\right)}{y_{n}-y_{0}}\right] d x \rightarrow \int_{a}^{b} D_{2} f\left(x, y_{0}\right) d x \quad \text { as } n \rightarrow \infty \text {. }
$$

A somewhat weaker but less clumsy form of this theorem is:

Suppose $f:[a, b] \times S \rightarrow \mathbb{R}$, where $S$ is an interval, and that for every point $y \in S$, the Riemann integral

$$
\phi(y)=\int_{a}^{b} f(x, y) d x
$$

exists. Suppose that for every $x \in[a, b]$ and for every $y \in S$, the partial derivative $D_{2} f(x, y)$ exists, and that the Riemann integral $\int_{a}^{b} D_{2} f(x, y) d x$ exists. Suppose finally that there exists a number $K$, such that for all $x \in[a, b]$, and $y \in S$, we have $\left|D_{2} f(x, y)\right| \leqslant K$. Then for every $y \in S$, we have

$$
\phi^{\prime}(y)=\int_{a}^{b} D_{2} f(x, y) d x .
$$

The useful analogues of this theorem for improper Riemann integrals can be deduced almost as simply, using an obvious "dominated convergence" analogue of the bounded convergence theorem which would apply to improper Riemann integrals. As an example of the sort of result that can be obtained, we cite the following:

THEOREM ON DifFERENTIATING AN IMPROPER INTEGRAL UNDER THE INTEGRAL Sign. Suppose $-\infty<a<b \leqslant \infty, S$ is an interval, and that $f:[a, b) \times S \rightarrow \mathbb{R}$. Suppose that for every point $x \in[a, b)$, the function $f(x, \cdot)$ is differentiable on $S$, and that for every point $y \in S$, the functions $f(\cdot, y)$ and $D_{2} f(\cdot, y)$ are improper Riemann integrable on $[a, b)$, and suppose finally that there exists an improper Riemann integrable function $g$ on $[a, b)$ such that for all $x \in[a, b)$ and $y \in S$, we have $\left|D_{2} f(x, y)\right| \leqslant g(x)$. 
Then if we define

$$
\phi(y)=\int_{a}^{\rightarrow b} f(x, y) d x
$$

for all $y \in S$, we have

$$
\phi^{\prime}(y)=\int_{a}^{\rightarrow b} D_{2} f(x, y) d x
$$

at every point $y \in S$.

Inversion of repeated integrals. The sharpest known result on inversion of iterated Riemann integrals is the elegant result that was proved in 1913 by G. Fichtenholz. We shall state three versions of Fichtenholz's theorem. The first of these is the easiest to prove, the second is the best possible result for Riemann integrable functions, and the third form is the ultimate theorem on the inversion of iterated integrals for a bounded function defined on a rectangle. In this third form of the theorem, we see that the theorem remains true even if some of the integrals are only assumed to be Lebesgue integrals.

Fichtenholz's TheOREM ON INVERSION OF ITERATED INTEGRALS First Form. Suppose $f$ is a bounded function on the rectangle $[a, b] \times[c, d]$. Then the identity

$$
\int_{a}^{b} \int_{c}^{d} f(x, y) d y d x=\int_{c}^{d} \int_{a}^{b} f(x, y) d x d y
$$

will hold if both sides exist as repeated Riemann integrals.

SECOND Form. Suppose $f$ is a bounded function on the rectangle $[a, b] \times[c, d]$. Suppose that for every point $x \in[a, b]$, the function $f(x, \cdot)$ is Riemann integrable on $[c, d]$, and that for every point $y \in[c, d]$, the function $f(\cdot, y)$ is Riemann integrable on $[a, b]$. Then

(a) The function $\phi:[a, b] \rightarrow \mathbb{R}$ defined by $\phi(x)=\int_{c}^{d} f(x, y) d y$ for all $x \in[a, b]$, is Riemann integrable on $[a, b]$,

(b) The function $\psi:[c, d] \rightarrow \mathbb{R}$ defined by $\psi(y)=\int_{a}^{b} f(x, y) d x$ for all $y \in[c, d]$, is Riemann integrable on $[c, d]$,

(c) $\int_{a}^{b} \phi(x) d x=\int_{c}^{d} \psi(y) d y$, in other words,

$$
\int_{a}^{b} \int_{c}^{d} f(x, y) d y d x=\int_{c}^{d} \int_{a}^{b} f(x, y) d x d y .
$$

THIRD Form. Suppose $f$ is a bounded function on the rectangle $[a, b] \times[c, d]$. Suppose that for every point $x \in[a, b]$, the function $f(x, \cdot)$ is Riemann integrable on $[c, d]$, and that for every point $y \in[c, d]$, the function $f(\cdot, y)$ is Lebesgue measurable on $[a, b]$. Then

(a) The function $\phi:[a, b] \rightarrow \mathbb{R}$ defined by $\phi(x)=\int_{c}^{d} f(x, y) d y$ for all $x \in[a, b]$, is Lebesgue measurable on $[a, b]$,

(b) The function $\psi:[c, d] \rightarrow \mathbb{R}$ defined by $\psi(y)=\int_{a}^{b} f(x, y) d x$ for all $y \in[c, d]$, is Riemann integrable on $[c, d]$, 
(c) $\int_{a}^{b} \phi(x) d x=\int_{c}^{d} \psi(y) d y$, in other words,

$$
\int_{a}^{b} \int_{c}^{d} f(x, y) d y d x=\int_{c}^{d} \int_{a}^{b} f(x, y) d x d y .
$$

Proof of the first form. For each natural $n$, denote as $\mathscr{P}_{n}$ the regular $n$-partition of $[c, d]$. For $i=1, \ldots, n$, the $i$ th point of $\mathscr{P}_{n}$ is, of course, $c+i(d-c) / n$, but for simplicity, we shall denote this as $y_{n i}$. For each natural $n$ and $x \in[\mathrm{a}, \mathrm{b}]$, define

$$
\phi_{n}(x)=\sum_{i=1}^{n} f\left(x, y_{n i}\right)\left(y_{n i}-y_{n i-1}\right) .
$$

Since the function $f(x, \cdot)$ is Riemann integrable for every $x \in[a, b]$ and since $\left\|\mathscr{P}_{\mathrm{n}}\right\| \rightarrow 0$, it follows from Darboux's theorem that $\phi_{n}(x) \rightarrow \phi(x)$ for each $x \in[a, b]$. Since we have also assumed that $\phi$ is Riemann integrable on $[a, b]$, it follows from the bounded convergence theorem that

$$
\int_{a}^{b} \phi_{n}(x) d x \rightarrow \int_{a}^{b} \phi(x) d x=\int_{a}^{b} \int_{c}^{d} f(x, y) d y d x \quad \text { as } \quad n \rightarrow \infty .
$$

But for each $n$, we have

$$
\begin{aligned}
\int_{a}^{b} \phi_{n}(x) d x & =\int_{a}^{b} \sum_{i=1}^{n} f\left(x, y_{n i}\right)\left(y_{n i}-y_{n i-1}\right) d x \\
& =\sum_{i=1}^{n}\left[\int_{a}^{b} f\left(x, y_{n i}\right) d x\right]\left(y_{n i}-y_{n i-1}\right) \\
& =\sum_{i=1}^{n} \psi\left(y_{n i}\right)\left(y_{n i}-y_{n i-1}\right)
\end{aligned}
$$

and since $\psi$ is Riemann integrable on $[c, d]$, the latter expression approaches $\int_{c}^{d} \psi(y) d y$ as $n \rightarrow \infty$.

This shows that $\int_{a}^{b} \phi(x) d x=\int_{c}^{d} \psi(y) d y$ which is what we had to prove.

Proof of the second form. The difference between this second form of the theorem and the first form, is that the Riemann integrability of the functions $\phi$ and $\psi$ is now part of the conclusion. What we have to show therefore, is that $\phi$ and $\psi$ are automatically Riemann integrable on $[a, b]$ and $[c, d]$, respectively. As above, let $\mathscr{P}_{n}$ be the regular $n$-partition of $[c, d]$ for each natural $n$, and denote the $i$ th point of $\mathscr{P}_{n}$ as $y_{n i}$. To show that $\psi$ is Riemann integrable on $[c, d]$, we shall show that there is a number $L$ such that for every possible choice of numbers $t_{n i}$ in the intervals $\left[y_{n i-1}, y_{n i}\right]$ we have

$$
\sum_{i=1}^{n} \psi\left(t_{n i}\right)\left(y_{n i}-y_{n i-1}\right) \rightarrow L \text { as } n \rightarrow \infty .
$$

Let us look for the moment at one possible choice of the nunbers $t_{n i}$. For each 
natural $n$ and $x \in[a, b]$, define

$$
\phi_{n}(x)=\sum_{i=1}^{n} f\left(x, t_{n i}\right)\left(y_{n i}-y_{n i-1}\right) .
$$

Since the function $f(x, \cdot)$ is Riemann integrable for every $x \in[a, b]$ and since $\left\|\mathscr{P}_{n}\right\| \rightarrow 0$, it follows from Darboux's theorem that $\phi_{n}(x) \rightarrow \phi(x)$ for each $x \in[a, b]$. It, therefore, follows from the bounded convergence theorem that the sequence of integrals $\int_{a}^{b} \phi_{n}(x) d x$ converges. The limit of this sequence of integrals is obviously independent of the choice of numbers $t_{n i}$ for if $t_{n i}^{*}$ is another choice, and the functions $\phi_{n}^{*}$ are defined analogously by

$$
\phi_{n}^{*}(x)=\sum_{i=1}^{n} f\left(x, t_{n i}^{*}\right)\left(y_{n i}-y_{n i-1}\right) \text { for } x \in[a, b]
$$

then we also have $\phi_{n}^{*}(x) \rightarrow \phi(x)$ for all $x \in[a, b]$ and the bounded convergence theorem implies that $\int_{a}^{b}\left[\phi_{n}(x)-\phi_{n}^{*}(x)\right] d x \rightarrow 0$. Now for each $n$, we have

$$
\int_{a}^{b} \phi_{n}(x) d x=\sum_{i=1}^{n} \psi\left(t_{n i}\right)\left(y_{n i}-y_{n i-1}\right)
$$

and, therefore, the latter expression tends to a limit as required. This shows that $\psi$ is Riemann integrable on $[c, d]$. The proof that $\phi$ is Riemann integrable on $[a, b]$ is similar.

Proof of the third form. As in the proof of the second form, we need to show that $\psi$ is Riemann integrable on $[c, d]$. The proof we use now is similar to the one used before except that this time, we have to make use of the Lebesgue dominated convergence theorem. As before, denote as $\mathscr{P}_{n}$ the regular $n$-partition of $[c, d]$ and the $i$ th point of $\mathscr{P}_{n}$ as $y_{n i}$. We shall prove the theorem by showing that $\phi$ is measurable on $[a, b]$, and that for every possible choice of numbers $t_{n i}$ in the intervals $\left[y_{n i-1}, y_{n i}\right]$ for $n=1, \ldots$ and $i=1, \ldots, n$, we have

$$
\sum_{i=1}^{n} \psi\left(t_{n i}\right)\left(y_{n i}-y_{n i-1}\right) \rightarrow \int_{a}^{b} \phi(x) d x \text {. }
$$

Suppose then, that the numbers $t_{n i}$ have been chosen. For each natural $n$ and $x \in[a, b]$, define

$$
\phi_{n}(x)=\sum_{i=1}^{n} f\left(x, t_{n i}\right)\left(y_{n i}-y_{n i-1}\right),
$$

and notice that each function $\phi_{n}$, being a linear combination of Lebesgue measurable functions, is Lebesgue measurable on $[a, \mathrm{~b}]$. As above, it follows from the Riemann integrability of the functions $f(x, \cdot)$ that $\phi_{n}(x) \rightarrow \phi(x)$ for each $x \in$ $[a, b]$. Therefore $\phi$ is Lebesgue measurable on $[a, b]$ and it follows from the Lebesgue dominated convergence theorem that

$$
\int_{a}^{b} \phi_{n}(x) d x \rightarrow \int_{a}^{b} \phi(x) d x
$$


and the result follows as before from the identity

$$
\int_{a}^{b} \phi_{n}(x) d x=\sum_{i=1}^{n} \psi\left(t_{n i}\right)\left(y_{n i}-y_{n i-1}\right) .
$$

An interesting (and possibly surprising) feature of Fichtenholz's theorem is the fact that it makes no requirement of integrability of $f$ jointly in the two variables $x$ and $y$. The theorem is, therefore, quite different in character from Fubini's theorem and from the theorems on pages 111-114 of Buck [2] and those in Section 7.25 of Apostol [1]. As is well known, if the Continuum Hypothesis is assumed, then the analogue of Fichtenholz's theorem for Lebesgue integrals is not even true; see Rudin [5, page 152]. This means that the above requirement of Riemann integrability of the function with respect to at least one of its variables is really needed. Some further counterexamples may be found in Luxemburg [4], which also contains a significant generalization of Fichtenholz's theorem to some abstract theories of integration. But it should be mentioned that one of the examples cited by Luxemburg is incorrect, possibly a result of a misreading of Proposition $\mathrm{C}_{49}$ in Sierpiński [6]. Luxemburg cites the incorrect example as a counter example to the above third form of Fichtenholz's theorem.

\section{REFERENCES}

1. Tom Apostol, Mathematical Analysis, Second ed., Addison-Wesley Publishing Company, Reading, Massachusetts, 1974.

2. R. Creighton Buck, Advanced Calculus, McGraw-Hill Book Company, New York, 1965.

3. Jonathan W. Lewin, A truly elementary approach to the bounded convergence theorem, American Mathematical Monthly, 93 (1986) 395-397.

4. W. A. J. Luxemburg, The Abstract Riemann Integral and a Theorem of G. Fichtenholz on Equality of Repeated Riemann Integrals, IA and IB, Proc. Ned. Akad. Wetensch. Ser. A 64 (1961) 516-545 = Indag. Math., 23 (1961).

5. Walter Rudin, Real and Complex Analysis, McGraw-Hill Book Company, New York, 1974.

6. Sierpiński, W., Hypothèse du Continu, Warsaw (1934), second. ed., New York, 1956. 